\title{
MSECO-DEV: Application Development Process in Mobile Software Ecosystems
}

\author{
Awdren de L. Fontão \\ Institute of Computing \\ UFAM \\ Manaus - AM, Brazil \\ awdren@icomp.ufam.edu.br
}

\author{
Rodrigo Pereira dos Santos \\ PESC/COPPE \\ UFRJ \\ Rio de Janeiro - RJ, Brazil \\ rps@cos.ufrj.br
}

\author{
Jackson Feijó Filho \\ Institute of Technology \\ Development - INDT \\ Manaus - AM, Brazil \\ jackson.feijo@indt.org.br
}

\author{
Arilo Claudio Dias-Neto \\ Institute of Computing \\ UFAM \\ Manaus - AM, Brazil \\ arilo@icomp.ufam.edu.br
}

\begin{abstract}
In a Mobile Software Ecosystem (MSECO), the central organization (keystone), must restructure processes to aid external developers to produce mobile applications. The external developer helps the keystone to reach goals, such as growing number of mobile applications. However, there is no process in this context to support developers in the development aligned with the keystone's goals. This paper presents MSECO-DEV, a process to support external developers in reaching keystone's goals by developing mobile applications. MSECO-DEV comprises 8 activities, 7 artifacts, 8 recommendations, and 17 practices. Activities, recommendations, and practices were evaluated by 65 Brazilian developers (experts and novices). Such developers acted within the main MSECOs (Android, iOS and Windows Phone) to assess their benefits for the mobile applications development routine. As result, we stated that developers have difficulties to perform marketing activities, as well as to find materials that support development. Practices, activities, and recommendations were also evolved and adjusted for the definition of MSECO-DEV.
\end{abstract}

Keywords - Software Ecosystem; Mobile; Software Process.

\section{INTRODUCTION}

A MSECO comprises several elements, such as mobile applications, mobile applications store, users, keystone (i.e., central organization), and external developers [1]. Each element in the MSECO has an expected responsibility and performance that depend on the management activities performed by its keystone. The keystone needs to assure that the ecosystem keeps running and meets its goals, such as growing number of developers within the ecosystem, and of mobile applications available on the mobile application store [1].

In this context, the mobile application stores act as important MSECO instruments to make the entry of new developers and mobile applications easier. However, mobile application store and keystone cannot achieve their goals by themselves [1]. Fontão et al. [2] pointed out the following research opportunity: "how does the quality assurance strategies depend on the specific solutions proposed to each MSECO (e.g., practices to help it in running successful, and guidelines to develop mobile apps)?’”.

As an alternative to this scenario, the MSECOs have been investing in tools, forums, and support materials. Such strategy aims to create an effective relation between developers and the keystone [3]. In this context, there is a difficult task: balancing keystone's and external developer's expectations [3]. Even with the keystone's investment in the mobile development aid, few practices and processes have been defined to support the developer's work [1]. This situation leverages the need for customized development processes to sustain the development of high quality mobile applications that are able to remain competitive in the MSECO [4].

Based on this scenario, this paper presents a process to support mobile applications' developers aiming to reach keystone's goals, named MSECO-DEV. To define MSECODEV, we followed a methodology (detailed in Section III.A) that comprises: (1) execution of an observational study, (2) a systematic mapping study, (3) a first evaluation with expert developers, and (4) a second evaluation with novice developers. In addition to MSECO-DEV process itself, as an important contribution of this work, the methodology reported on the definition of the MSECO-DEV can serve as basis for new contributions in the software ecosystems research. This paper is organized as follows. Section II presents background. Section III describes MSECO-DEV and the methodology to build it. Section IV presents the planning, execution and results of the first survey conducted to evaluate the process with experienced developers in MSECO. Section V discusses procedures and results of the second survey conducted with novice developers to evaluate the activities and practices. Finally, Section VI concludes the paper with future work.

\section{DEVELOPERS IN MSECO}

The Developer eXperience (DX) consists of experiences related to all types of activities that a developer might encounter as part of their involvement in software development. We can mention as sources of DX: (a) infrastructure development: management and development tools, programming languages, libraries, platforms, processes, and methods; (b) perceptions about the work: respect and recognition; and (c) sense of contribution: alignment of developers' work and contributions regarding keystone's objectives and plans [6].

Concerning "infrastructure development", the keystone needs to undertake efforts to provide developers with a framework aiming to expand the MSECO frontiers. It can be achieved with guidelines to create new apps. From the 
perspective of "perceptions about the work", the developer needs to create and deliver apps that reach the best existing niches of users, and gain visibility into the app store based on the quality of apps. Finally, regarding "sense of contribution", the keystone has goals that can be increased by external developers as: (i) the number of ecosystem's apps, and (ii) the star ratings of apps published in the app store. However, keystones can hardly meet the entire demand of society only with their own internal structure. Within a MSECO, external developers can help organizations in maintaining their strategy since they work directly in solving this limitation [1].

In this scenario, developers rarely use any formal development processes and have no organized tracking of their development efforts [7]. Regarding the development processes in the context of MSECO, related work that offers insights into potential alternatives to the MSECO-DEV was not found [1]. As the app development become more complex, it will be essential to adapt and/or apply software engineering processes to ensure the construction of high-quality apps [7]. For this reason, there is a need for structuring processes for managing elements of an MSECO [5]. It involves all app development activities performed by a developer, once the MSECO context is dynamic and depends on the keystone goals [6].

\section{MSECO-DEV: APPLICATION DEVELOPMENT PROCESS IN MSECO}

In this section, we present the MSECO-DEV (Mobile Software Ecosystem - Development). This process aims to improve the perception of app development by structuring a process and evaluating benefits for developers (Figure 1).

\section{A. Methodology}

The methodology to define MSECO-DEV was based on five steps. Initially, an observational study was conducted through training sessions within the Windows Phone MSECO with 716 developers where the keystone's goals were driven by the growing number of downloads, apps, and developers, as detailed in [3]. This study helped us to propose MSECO Skill methodology, and to identify activities of app development in the MSECO context. With the application of MSECO Skill, the Windows Phone MSECO keystone (Microsoft) expanded the quantity and quality of mobile apps. This study concluded that there is a lack of app development approaches in the context of a MSECO to support external developers' activities.

After running the observational study, a systematic mapping study of the technical literature was conducted in order to extract processes, benefits, characteristics, and areas studied in MSECO [1]. This mapping study pointed out the lack of approaches, methodologies, processes, and tools to support MSECO. We also analyzed the selected studies to extract recommendations (e.g., guidelines to implement activities) and practices (e.g., exercises to achieve concrete results regarding the keystone's goals) that might be associated with the MSECO-DEV activities.
The results of the observational study [3] jointly with the results of the systematic mapping study [1] helped us in the construction of $\boldsymbol{M S E C O}-\boldsymbol{D E} \boldsymbol{V}$, an app development process that encompasses the developer's activities within an MSECO, including recommendations and practices associated to their activities. This approach is presented in the next sections. In the Sections IV and V, we discuss the last two steps of the methodology we followed to build MSECO-DEV.

\section{B. Definition of MSECO-DEV}

In a MSECO, a keystone needs to support communication and coordination of external developers throughout processes' phases, as well as to investigate the impacts of different practices in project planning, integration and development standards [7]. In MSECO-DEV, activities provide information and allow the development of an app aligned with keystone's goals. The notation used to describe the MSECO-DEV is based on SPEM language ${ }^{1}$. The artifact generated at the end of this process should be a publishable file packaged built from standards provided in the MSECO and must be supported by its platform. This artifact will be available in the app store, and it can be embedded in a user's device.

For each activity, a recommendation consists of a guide to perform an activity to which it has been associated with. In turn, for each recommendation, exercises to achieve concrete results regarding the keystone's goals were associated, labeled as practices. The practices are only associated with the activities prior to submission of an app to the store. In this section, we present a set of 8 recommendations (one per activity) and 14 practices which compose the MSECO-DEV process (Table 1). Once MSECO-DEV recommendations and practices were built, we evaluated them with developers of different MSECOs. Thus, we planned and conducted two surveys (Sections IV and V).

\section{FIRST SURVEY WITH EXPERIENCED DEVELOPERS}

This section presents a survey planned and executed with the goal of analyze recommendations and practices that compose the MSECO-DEV with the purpose of characterizing with respect to their usefulness from the point of view of experienced developers in the context of app development activities in MSECO.

\section{A. Survey's Research Questions and Instrumentation}

The research questions (RQs) and their related metrics are described in the Table 2. As instrumentation, we prepared a questionnaire to evaluate the relationship between practices and recommendations with the activities of the MSECO-DEV through the assertion: "Is [recommendation/practice] related to the activity [activity] as in your routine?". 5-points Likert scale was used offering the following options: 1. Totally Agree; 2. Partially Agree; 3. I do not know (neutral); 4. Partially Disagree; and 5. Totally Disagree.

\footnotetext{
${ }^{1}$ http://www.omg.org/spec/SPEM/
} 
Table 1. Activities, Recommendations and Practices. [Activity 1] to define the app idea (scope), taking into account the devices, and also APIs and SDKs used in the MSECO.

Recommendation: Consider how hardware and operating system software features (e.g. screen navigation control) can affect app development.

(P1) Use support materials when answering questions about the correct definition of the developer's idea;

(P2) Define scope to confirm the viability of the idea;

(P3) Analyze a set of apps as well as non-existing apps in the MSECO;

(P4) Analyze a set of successful apps within App Store;

(P5) Identify niche opportunities for app development, i.e., society needs;

(P6) Analyze non-successful apps within the app store.

[Activity 2] to prepare the app's marketing material in order to help developers to create user interface compatible with the platform.

Recommendation: Evolve developer's idea to prepare marketing material for an app; images and icons must follow standards;

(P7) Prepare apps based on support to generate marketing materials that can be used within the app store and in other information channels.

[Activity 3] to develop the app through tools provided by ecosystem. At the end of this activity, there is a binary.

Recommendation: Use design standards for the app development.

(P8) Use design patterns in app development;

(P9) Apply app store's acceptance criteria in the app development;

(P10) Use platform interface design guides to ensure the app identity;

(P11) Develop an app driven by user experience.

[Activity 4] to analyze binary against the quality criteria of the app store.

Recommendation: Generate a document with the results of the analysis.

(P12) Use tools to enable the verification of the binary;

(P13) Use binary validation tools;

[Activity 5] to analyze marketing package according to the guidelines.

Recommendation: Include images of app screens into the package.

(P14) Use a checklist to ensure the completeness of the generated marketing.

[Activity 6] to submit the binary and the marketing package to the developer central portal.

Recommendation: Be aware of submission rules that can be found in the support material.

[Activity 7] to monitor the app acceptance status in the store.

Recommendation: Monitor apps via e-mails or check the developer central portal.

[Activity 8] to monitor the reports published in the store.

Recommendation: Consider users' comments and reviews related to app

evolution, and release updates in order to add new features.
Table 2. First Survey - Research Questions and Metrics.

RQ1. What are the recommendations to the app development activities within an MSECO?

Metric: the list of recommendations of MSECO-DEV process that are effectively useful in the app development activities (recommendations consolidated from the initial set, or added by participants of the study).

RQ2. What are the practices in the app development within an MSECO?

Metric: the list of practices of MSECO-DEV process that are effectively useful in the app development activities (practices consolidated from the initial

set, or added by the participants of the study).

\section{B. Participants' Profile}

The distribution of these developers regarding their experience in app development is the following: 22 (individually), 8 (as a part of a team, startup, or only a team) and 10 (as a part of a team in industry). The participants were asked to inform in which MSECOs they work (or already worked). The results obtained for the three major MSECOs are: 40\% (Windows Phone), 30\% (Android), 13\% (Other 'dead' Nokia's MSECOs), 10\% (Symbian), and 7\% (iOS). As mentioned, they could act in more than one MSECO.

\section{Analysis of the Recomendations}

Regarding the recommendations, Table 3 presents the number of responses for each option available in the questionnaire: total agreement $(T A)$, partial agreement $(P A)$, if the participant had no formal opinion $(N O)$, partial disagreement $(D P)$, or total disagreement $(T D)$. In addition, there is a column in Table 3 labeled Disagreement Level $(D L)$ to represent the percentage of negative responses $(P D$ or $T D)$. As highlighted in Table 3, three recommendations (38\%) did not reach $D L=0 \%$. It means that five recommendations $(62 \%)$ were confirmed by participants, that is, $D L=0 \%$. The recommendations with some level of disagreement are associated to Activities 2, 4 and 5.

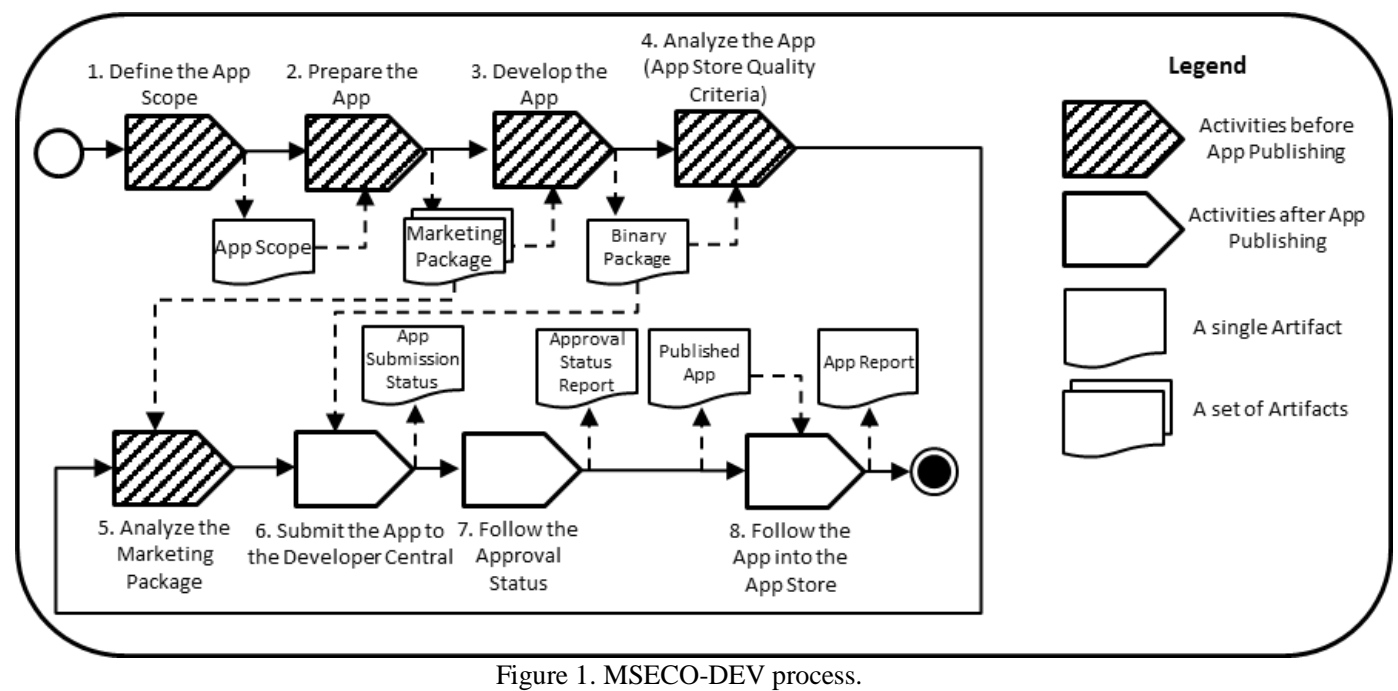


The disagreement regarding the recommendation associated to Activity 2 might be caused because it is part of an initial activity in the process, according to a developer: "It is a bit rash to think on how to sell something that you do not have yet; many things and ideas will possibly change along the development".

Table 3. Analysis of Practices and Recommendations.

\begin{tabular}{|c|c|c|c|c|c|c|c|}
\hline Activity & Practice & TA & PA & NO & PD & TD & DL \\
\hline \multirow{7}{*}{1} & Recommendation & 29 & 4 & 0 & 0 & 0 & $0 \%$ \\
\hline & P1 & 26 & 6 & 1 & 0 & 0 & $0 \%$ \\
\hline & $\mathbf{P 2}$ & 26 & 6 & 1 & 0 & 0 & $0 \%$ \\
\hline & P3 & 19 & 11 & 3 & 0 & 0 & $0 \%$ \\
\hline & P4 & 19 & 11 & 2 & 1 & 0 & $3 \%$ \\
\hline & P5 & 22 & 9 & 2 & 0 & 0 & $0 \%$ \\
\hline & P6 & 20 & 12 & 1 & 0 & 0 & $0 \%$ \\
\hline \multirow{2}{*}{2} & Recommendation & 18 & 12 & 0 & 3 & 0 & $9 \%$ \\
\hline & P7 & 20 & 10 & 2 & 0 & 1 & $3 \%$ \\
\hline \multirow{5}{*}{3} & Recommendation & 16 & 13 & 4 & 0 & 0 & $0 \%$ \\
\hline & P8 & 19 & 13 & 1 & 0 & 0 & $0 \%$ \\
\hline & P9 & 28 & 4 & 1 & 0 & 0 & $0 \%$ \\
\hline & P10 & 19 & 11 & 3 & 0 & 0 & $0 \%$ \\
\hline & P11 & 29 & 3 & 0 & 1 & 0 & $3 \%$ \\
\hline \multirow{3}{*}{4} & Recommendation & 20 & 8 & 2 & 2 & 1 & $9 \%$ \\
\hline & P12 & 21 & 9 & 2 & 0 & 1 & $3 \%$ \\
\hline & P13 & 19 & 9 & 5 & 0 & 0 & $0 \%$ \\
\hline \multirow{2}{*}{5} & Recommendation & 20 & 8 & 2 & 2 & 1 & $9 \%$ \\
\hline & P14 & 24 & 8 & 1 & 0 & 0 & $0 \%$ \\
\hline 6 & Recommendation & 29 & 4 & 0 & 0 & 0 & $0 \%$ \\
\hline 7 & Recommendation & 29 & 2 & 2 & 0 & 0 & $0 \%$ \\
\hline 8 & Recommendation & 30 & 3 & 0 & 0 & 0 & $0 \%$ \\
\hline
\end{tabular}

In the Activity 4, there was a disagreement in the recommendation reported by a developer as follows: "It is not necessary to generate a document summarizing the result of the analysis". In the recommendation associated with Activity 5, the disagreement was observed on the marketing package and images, since there is a need for preparation of promotional video and marketing strategies - and it is a complex task in the application development.

\section{Analysis of the Practices}

As highlighted in Table 3, four practices (29\%) did not reach $D L=0 \%$. It means that 10 practices $(71 \%)$ were confirmed by participants, that is, $D L=0 \%$. However, the participants have provided some suggestions for the adjustment of the 4 practices.

The Practice P4 related to the Activity 1 got $D L$ greater that $0 \%$. With regard to this practice, participants commented: "The analysis of apps (successful or not) can be seen only as an indication, but should not be a fundamental item".

The Practice P7 related to Activity 2 obtained some level of disagreement. The disagreement seemed to be associated with the fact that, perhaps, it is not the best moment to think about marketing (or maybe the developer is not the best role to prepare strategies and marketing).

Regarding the Activity 3, the Practice P11 had some level of disagreement. It was reported that "the usability concern is a requirement. The use of templates - wireframes, prototypes, and mockups, for example - also serves for this purpose".

In the practices associated with Activity 4, a participant disagreed with the Practice P12 because he/she "understands the operation of binary validation tools as part of the app's validation and also as part of the analysis of the development feasibility".

\section{E. New Suggested Practices}

During the study, participants suggested three new practices, described as NewP<Sequence Number> - Activity <number>, which was included in the MSECO-DEV (Table 4).

Table 4. First Survey - New Suggests Practices.

NewP1 - Activity 1: Pay attention to the gaps of similar apps, and to the comments of frustrated users, in order to be able to meet the ecosystem's expectations

NewP2 - Activity 1: Evaluate the effort and complexity of using an app, if it uses or requires third-party APIs.

NewP3 - Activity 2: Prepare the app to offer a consistent user experience, since users will always recommend others with the apps they like.

\section{F. Threats to Validity}

Conclusion validity: accomplished through simple demonstration of presence (or not) of recommendations and practices stated in the MSECO-DEV.

Internal validity: for this study, we proposed to select developers who work in the main MSECOs. Thus, we assumed that they are representative for the population of MSECO developers.

Constructo validity: the study is characterized by applicability analysis of the recommendations and practices associated with the MSECO-DEV activities with respect to the current activities required by the app development in MSECO.

External validity: as mentioned in Internal Validity, the participants act in the main MSECOs. However, new studies could be performed with more developers.

\section{SECOND SurVey WITH NOVICE DEVELOPERS}

The goal of this second survey was to analyze a subset of practices and activities that compose the MSECO-DEV with the purpose of characterizing with respect to their usefulness and applicability from the point of view of novice developers in the context of app development activities in MSECO.

\section{A. Research Questions and Instrumentation}

The RQs and related metrics to help us to answer them are presented in Table 5. 
Table 5. Second Survey - Research Questions and Metrics. RQ1. Are the activities that compose MSECO-DEV applied by developers before the submission of an app to the app store?

Metric: the list of MSECO-DEV activities applied in the app development.

RQ2. Are the practices related to the activities that compose MSECO-DEV

useful and applicable by developers before the submission of an app to the app store?

Metric: the list of MSECO-DEV practices that are useful and applied in app development.

As the first survey, a questionnaire was prepared to evaluate the usefulness and applicability of the activities and practices, and the existing relations among them.

\section{B. Participants' Profile}

We invited novice developers who are undergraduate or graduate students attending a Mobile Applications Engineering course. During the course, developers had contact with professionals from academia and industry covering topics necessary for the development of apps, such as monetization, design, testing, user experience, tools etc. At the end of the course, they developed an app applying the concepts they learned.

In total, 32 developers participated in this study. When were asked about which MSECOs they work or worked, the results were: $32(100 \%)$ work/worked in the Android, $3(9 \%)$ work/worked in the iOS, and 5 (16\%) work/worked in the Windows Phone. Participants did not use MSECO-DEV to develop the app during the course.

\section{Analysis of Activities}

As mentioned in the purpose of the study, the participants have reviewed the activities that compose the pre-publication stage. In other words, the developers did not submit their mobile apps and they did not have the experience in evaluating the remainder activities. The following activities presented in Section III.B were evaluated: 1 - Define the App Scope, 2 Prepare the App, 3 - Develop the App, 4 - Analyze the App (App Store Quality Criteria), and 5 - Analyze the Marketing Package.

Each participant was asked to inform the activities they performed during the development of their projects (FP - Fully Performed, or PP - Partially Performed), or whether he/she did not perform some of them (NP - Not Performed). Table 6 shows the number of responses for each activity. From this data, it was possible to observe two main results. 19 developers $(59 \%)$ did not analyze the mobile applications against the app store criteria after the development (Activity 4). Moreover, 24 developers $(75 \%)$ did not analyze the marketing materials prepared for the app (Activity 5).

Regarding the Activity 4, the main critical comments were related to: 1) the app is still under development; 2) the lack of experience; 3) there was no specific time to perform a full analysis of the quality criteria; 4) another team should perform this activity; 5) there was no interest in making the app available on the app store; and 6) the lack of knowledge of the activity.

Regarding Activity 5, the developers listed as reasons to not execute it: 1) the lack of support and technical material; 2) the lack of time; 3 ) the development was not completed yet; and 4) no focus on providing the mobile application to the app store.

\section{Analysis of the Practices}

Concerning the practices, participants answered the questions selecting options from a 4-scale of usefulness/applicability: a) useful and already applied (U/A); b) useful and not applied yet $(U / N A)$; c) not useful and already applied (NU/A); d) not useful and not applied yet (NU/NA). Table 6 presents the participants' responses to each practice. After analyzing specifically the practices in which there is a great number of answers useful and not applied yet, we identified six practices as highlighted in Table 6 - all included in the following analysis.

Table 6. Usefulness and Applicability of Practices.

\begin{tabular}{|c|c|c|c|c|c|c|c|c|}
\hline Activity & FP & PP & NP & Practice & U/A & U/NA & NU/A & NU/NA \\
\hline \multirow{8}{*}{1} & \multirow{8}{*}{15} & \multirow{8}{*}{17} & \multirow{8}{*}{0} & $P 1$ & 25 & 6 & 1 & 0 \\
\hline & & & & $P 2$ & 31 & 1 & 0 & 0 \\
\hline & & & & P3 & 27 & 4 & 0 & 1 \\
\hline & & & & $P 4$ & 22 & 10 & 0 & 0 \\
\hline & & & & P5 & 19 & 13 & 0 & 0 \\
\hline & & & & P6 & 11 & 18 & 1 & 2 \\
\hline & & & & NewP1 & 19 & 13 & 0 & 0 \\
\hline & & & & NewP2 & 15 & 13 & 0 & 4 \\
\hline \multirow{2}{*}{2} & \multirow{2}{*}{14} & \multirow{2}{*}{15} & \multirow{2}{*}{3} & P7 & 5 & 22 & 2 & 3 \\
\hline & & & & NewP3 & 21 & 11 & 0 & 0 \\
\hline \multirow{4}{*}{3} & \multirow{4}{*}{31} & \multirow{4}{*}{1} & \multirow{4}{*}{0} & P8 & 23 & 9 & 0 & 0 \\
\hline & & & & $P 9$ & 7 & 25 & 0 & 0 \\
\hline & & & & $P 10$ & 22 & 10 & 0 & 0 \\
\hline & & & & P11 & 27 & 5 & 0 & 0 \\
\hline \multirow{2}{*}{4} & \multirow{2}{*}{5} & \multirow{2}{*}{8} & \multirow{2}{*}{19} & $P 12$ & 6 & 25 & 0 & 1 \\
\hline & & & & $P 13$ & 3 & 28 & 0 & 1 \\
\hline 5 & 4 & 4 & 24 & P14 & 2 & 30 & 0 & 0 \\
\hline
\end{tabular}

In the Activity 1, P6 was not applied by 18 (56\%) participants. A participant commented that: "Our team should consider enough time to check the feasibility of our app".

About P7, even considering this practice useful, 22 participants $(69 \%)$ did not implement it in the app development. About this practice, a participant gave the following feedback: “The 'first impression' of the user is always important. An app gets 'fame' with the comments from other users".

For P9, associated with Activity 3, 25 participants (78\%) did not apply it even considering it useful. The feedback that summarizes the reason the practice has not been applied is: "I knew these criteria but I did not know where to find them".

The two practices of Activity 4, P12 and P13, have been evaluated respectively as useful, but they were not applied by 25 (78\%) and 28 (88\%) participants, respectively. In respect to P12, a participant commented that: "I did not know where the tool 
was and even how to use it in practice". About P13, an answer was: "As we had no idea on how to submit the app to the store, we did not perform the tests as frequently as we should do".

Finally, in the Activity 5 (before the submission of the app to the store), P14 was evaluated by 30 participants (94\%) as useful, but they never applied it. A feedback from this evaluation is the following: "I did not analyze the lack of knowledge of the marketing guides for my app".

Even considered useful, this subset of practices should be applied again after an adjustment in the sequence in which they appear. Therefore, those practices should appear during the development, or prior to development. The practices can be adjusted in activities that define the scope of the app and prepare initial marketing packages for app.

\section{E. Threats to Validity}

Conclusion validity: accomplished through simple demonstration of presence (or not) of activities and practices of the MSECO-DEV.

Internal validity: for this study, we proposed to select developers who participate in MSECOs. Thus, we assumed that they are representative for the population.

Constructo validity: the study is characterized by analysis the of activities' sequence and practices, as well as the description of practices and activities and the association among them of the development process with respect to activities and practices necessary for the development of apps in MSECO.

External validity: Participants can be considered as a sample of the population once they received specific training sessions for the development of apps.

\section{CONCLUSION AND FUTURE WORK}

In the new business model raised from mobile platforms, even with the keystone's investment in the development support, few practices and processes have been defined to support the developer's work, as confirmed in [1]. Another point is that the keystone needs to expand the MSECO through the growing number of apps of good quality (star rating of apps) to attract more users. Due to this fact, the external developers - outside the keystone organization - need to develop apps that help to reach the keystone's goals.
In this scenario, we presented a process named MSECODEV that aims to support the developer in the construction of an app in the MSECO context. As an important contribution of this work, we can mention the set of activities, recommendations, and practices of the MSECO-DEV, that were defined and evaluated based on experimental studies.

As future work, we propose: (a) to perform case studies with at least two MSECOs in order to apply MSECO-DEV; (b) investigate other processes in MSECO, such as orchestration, which is focused on the keystone; (c) analyze the sources of Developer eXperience (DX) involved in app development process adopted by MSECOs; and (d) examine how an MSECO's processes impact the health of the ecosystem, i.e., the ability of a MSECO to survive the disruptions (e.g., developers escape) and remain productive.

\section{ACKNOWLEDGMENT}

The authors would like to thank FAPEAM, CNPq, CAPES (Proc. no. BEX 0204/14-5), and INDT for the financial support.

\section{REFERENCES}

[1] A. Fontão, R. P. Santos, A. C. Dias-Neto. 2015. Mobile Software Ecosystem (MSECO): A Systematic Mapping Study. In Proceedings of the 39 th Annual International Computers, Software \& Applications Conference (COMPSAC), Taichung, pp. 653-658.

[2] A. Fontão, R. P. Santos, A. C. Dias-Neto. 2015. Research Opportunities for Mobile Software Ecosystems. In Proceedings of the 9th Workshop on Distributed Software Development, Software Ecosystems and Systems-ofSystems (WDES), Belo Horizonte, pp. 97-98.

[3] A. Fontão, B. Bonifácio, A. Dias-Neto, A. Bezerra, R. P. Santos. 2014 MSECO Skill: Construção de Competências de Desenvolvedores em Ecossistemas de Software Móvel. In Proceedings of the 17th Iberoamerican Conference on Software Engineering (CIbSE), Pucón, pp. 81-94.

[4] L. Corral, A. Sillitti, G. Succi. 2013. Software development processes for mobile systems: Is agile really taking over the business? In Proceedings of the 1st International Workshop on the Engineering of Mobile-Enabled Systems (MOBS), San Francisco, pp. 19-24.

[5] S. Lim, P. Bentley, N. Kanakam, F. Ishikawa, S. Honiden. 2015. Investigating Country Differences in Mobile App User Behavior and Challenges for Software Engineering. IEEE Transactions on Software Engineering 41(1):40-64.

[6] F. Fagerholm, J. Münch. 2012. Developer experience: Concept and definition. In Proceeding of the International Conference on Software and System Process (ICSSP), Zurich, pp. 73-77.

[7] I. Wasserman. 2010. Software engineering issues for mobile application development. In Proceedings of the FSE/SDP Workshop on Future of Software Engineering Research (FoSER '10), Santa Fe, pp. 397-400. 